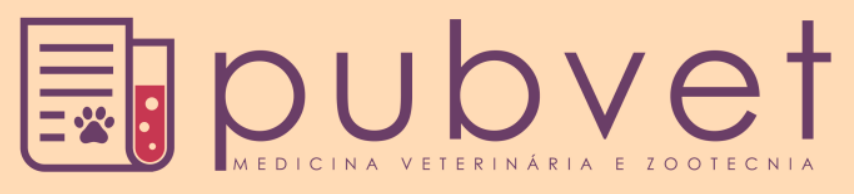

https://doi.org/10.31533/pubvet.v12n9a162.1-4

\title{
Infestação parasitária por Spirura sp. em Callithrix jacchus (Linnaeus, 1758): relato de caso
}

\author{
Brunna Muniz Rodrigues Falcão ${ }^{2 *} \bullet$, Débora Vitória Fernandes de Araújo ${ }^{1 \bullet}$, Joyce \\ Galvão de $\operatorname{Souza}^{2}{ }^{\circ}$, Artur da Nóbrega Carreiro ${ }^{\circ}{ }^{\circ}$, Jaqueline Bianque de Oliveira ${ }^{3}{ }^{\circ}$, \\ Stephenson Hallison Formiga Abrantes ${ }^{4}{ }^{\circ}$, José Rômulo Soares dos Santos ${ }^{5}{ }^{\circ}$, Danilo \\ José Ayres de Menezes ${ }^{6}{ }^{\circ}$, Gildenor Xavier Medeiros ${ }^{7}{ }^{\circ}$ \footnotetext{
${ }^{2}$ Aluno (a) do Programa de Pós-graduação em Medicina Veterinária, Universidade Federal de Campina Grande, Patos, PB, Brasil ${ }^{3}$ Professora da Universidade Federal Rural de Pernambuco, Departamento de Biologia, Recife, PE, Brasil

4 Professor da Universidade Federal de Campina Grande, Unidade Acadêmica de Ciências Biológicas, Patos, PB, Brasil

${ }^{5}$ Doutor em Medicina Veterinária, Técnico do Laboratório de Anatomia Veterinária, Universidade Federal da Paraíba, PB, Brasil

${ }^{6}$ Professor da Universidade Federal do Rio Grande do Norte, Departamento de Morfologia, Natal, RN, Brasil

'Professor da Universidade Federal de Campina Grande, Unidade Acadêmica de Medicina Veterinária, Patos, PB, Brasil, E-mail: gildenorxavier@ gmail.com

*Autor de correspondência, E-mail:brunnamrfalcao@hotmail.com
} \\ ${ }^{l}$ Aluna de Graduação, Universidade Federal de Campina Grande, Unidade Acadêmica de Medicina Veterinária, Patos, PB, Brasil,
}

RESUMO. O conhecimento de parasitos gastrintestinais em primatas é importante para o manejo da população de macacos e para a manutenção da saúde das pessoas, pois são animais comuns em cativeiro. O estudo relata um caso de parasitismo por Spirura sp. em um sagui-de-tufos-brancos adulto encontrado na região da Zona da Mata no estado da Paraíba. Foram identificados Spirura sp.na porção final do esôfago de um cadáver de sagui que foi doado para estudo anatômico pelo Centro de Triagem de Animais Silvestres da Paraíba.

Palavras chave: nematoda, parasito, sagui-de-tufos-brancos

\section{Parasitic infestation by Spirura sp. in Callithrix jacchus (Linnaeus, 1758): case report}

\begin{abstract}
The knowledge of gastrointestinal parasites in primates is important for the management of the population of monkeys and for the maintenance of the health of the people, since they are common animals in captivity. The study reports a case of parasitism by Spirura sp. in an adult Common marmoset found in the Zona da Mata region of Paraíba state. Spirura sp. was identified in the final portion of the esophagus of a Sagittarian corpse that was donated for anatomical study by the Paraíba Wild Animal Triage Center.
\end{abstract}

Keywords: nematoda, parasite, common marmoset

\section{Infestación parasitaria por Spirura sp. en Callithrix jacchus (Linnaeus, 1758): reporte de un caso}

RESUMEN. El conocimiento de parásitos gastrointestinales em primates es importante para el manejo de la población de monos y para el mantenimiento de la salud de las personas, pues son animales comunes en cautiverio. El estudio relata un caso de parasitismo por Spirura sp. en un mono [Callithrix jacchus (Linnaeus, 1758)] adulto encontrado em la región de la Zona de la Mata em el estado de Paraíba. Se identificaron Spirura sp. en la porción final del esófago de un cadáver de mono de mechones blancos que fue donado para estudio anatómico por el Centro de Clasificación de Animales Silvestres de Paraíba.

Palabras clave: nematodo, parásito, mono blanco de mechones blancos 


\section{Introdução}

Os saguis-de-tufos-brancos (Callithrix jacchus) pertencem a ordem Primates e a família Callitrichidae (Rylands, 2012), sendo os mais conhecidos e comuns na adaptação ao cativeiro, aspecto que estimula a captura clandestina destes animais. O Centro de Triagem de Animais Silvestres do Instituto Brasileiro do Meio Ambiente e dos Recursos Naturais Renováveis do Estado da Paraíba (CETAS/IBAMA/PB) recebe com frequência saguis apreendidos pelos agentes do Instituto Brasileiro do Meio Ambiente e dos Recursos Naturais Renováveis (IBAMA). Muitos saguis apreendidos eram mantidos em recintos inadequados e muitos chegam ao CETAS/ IBAMA/PB com ferimentos, desnutridos e com diversas doenças virais, bacterianas e parasitárias.

$\mathrm{O}$ estudo sobre parasitos gastrintestinais em primatas mantidos em cativeiros é importante para o manejo da população de macacos e para a manutenção da saúde das pessoas que trabalham com esses animais, pois muitos destes parasitos são potentes causadores de zoonoses. Também é relevante verificar se esses animais se comportam como transmissores de parasitas para o meio ambiente por estarem infectados (Brack, 2012). As infecções e as parasitoses estão diretamente relacionadas com as condições de vida do hospedeiro e do ambiente onde estes vivem. Os parasitos gastrintestinais são mais prevalentes em locais onde não há boas condições de higiene, principalmente com a água e os alimentos consumidos (Carmo \& Salgado, 2009).
Pretende-se, dessa forma, contribuir com pesquisas e a manutenção desses espécimes em cativeiro, assim como indiretamente na conservação desses animais de vida livre, fazendo reconhecimento de parasitas em animais de vida livre, pois eles causam doenças também pulmonares, cardiológicas, enterites, gastrites e intussuscepção intestinal, entre parasitas que possui risco zoonótico. Este trabalho relata um caso de parasitismo por Spirura sp. em um saguide-tufos-brancos adulto encontrado na região da Zona da Mata no estado da Paraíba.

\section{Relato de caso}

Foram encontrados parasitas do gênero Spirura sp. na porção final do esôfago de um cadáver de sagui-de-tufos-brancos doado para estudo anatômico pelo Centro de Triagem de Animais Silvestres da Paraíba (CETAS- PB) (Figura 1).

Os exemplares do parasito foram encaminhados ao Laboratório de Pesquisas Morfológicas da Universidade Federal de Campina Grande. Coletou-se o nematoide com pinça anatômica sem dente e em seguida lavou-se os parasitos com água destilada para fixação em formaldeído a $10 \%$ e foram enviados para identificação no Laboratório de Parasitologia no Departamento de Biologia da Universidade Federal Rural de Pernambuco (UFRPE). Para ser feita a identificação do gênero Spirura sp. deve se observar caraterísticas morfológicas, como a presença de uma ou duas corcovas ou bossas ventrais, segundo a metodologia de Vicente et al. (1997) e de Anderson et al. (2009).
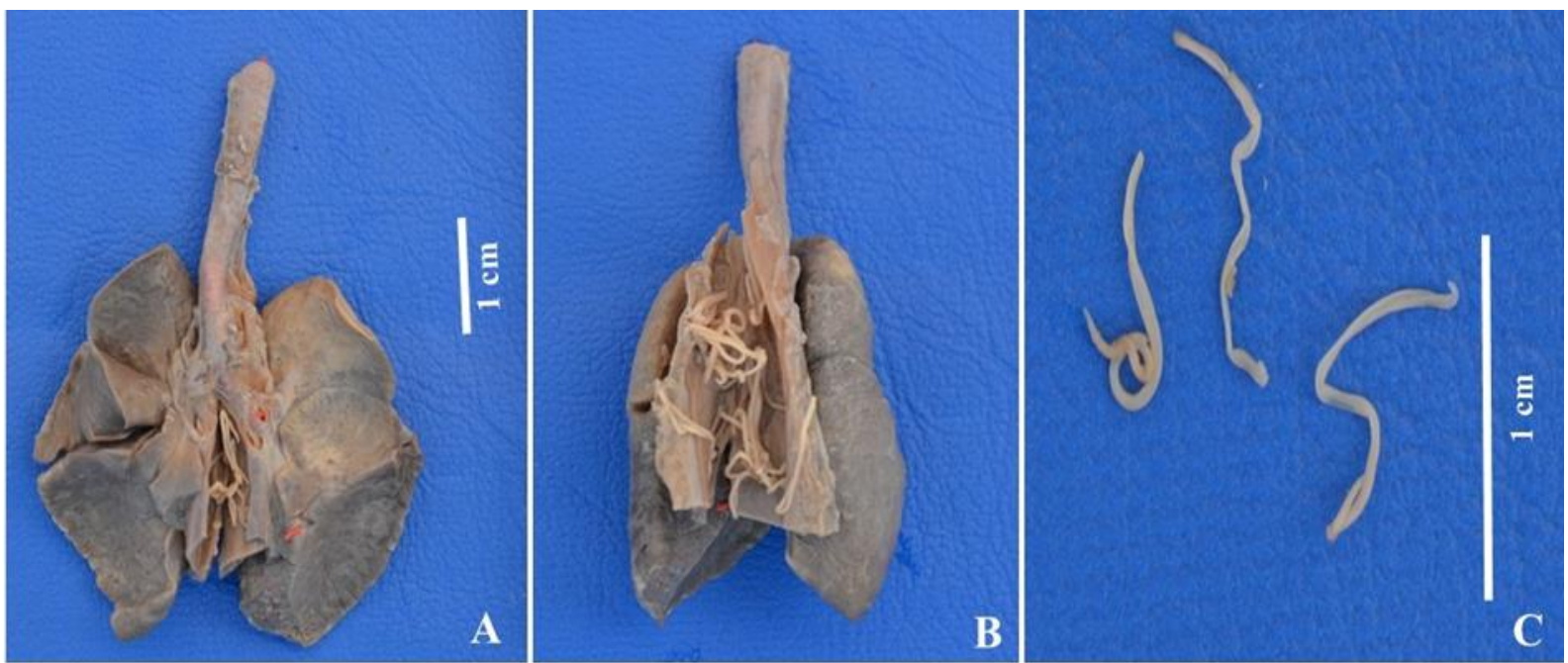

Figura 1. A) e B) Esôfago de um sagui-de-tufos-branco com parasitas da espécie Spirura, C) Spirura sp. encontrados em saguide-tufos-branco. 
Com auxílio de uma lupa estereoscópica, a identificação do parasito foi confirmada pelas características descritas para o gênero Spirura sp., apresentando a parte posterior do corpo acentuadamente mais espessa que a anterior, moderadamente espiralada, sem a presença de asa cervical, com a boca prolongada dorso ventralmente, cercada por um prolongamento quitinizado da cápsula bucal à qual está subvertida e densa dorsal e ventralmente, seguido por dois pequenos lábios trilobados e sem dentes em sua superfície interna. $\mathrm{O}$ envoltório bucal mostrou-se largo e cilíndrico quando visto lateralmente e muito comprimido em forma de funil quando visto dorsoventral. Apresentou esôfago muito extenso, cilíndrico, dividido em duas partes sem definição: uma anterior muito breve e uma posterior longa, parcialmente prolongada.

\section{Discussão}

A maior parte dos parasitos de primatas humanos e não humanos são nematoides. Nematoda é um filo antigo e biologicamente diverso de animais em constantes mudanças. Eles variam em tamanho de $0,2 \mathrm{~mm}$ a mais de $6 \mathrm{~m} \mathrm{e}$ podem ser encontrados na maioria dos habitats, inclusive dentro de animais e plantas hospedeiras, além de exibirem uma ampla gama de relações com outras espécies. O parasitismo é um modo de vida comum e uma grande proporção de espécies de nematoides podem ser parasitos. A relação parasito-hospedeiro pode ser classificada em diferentes tipos de relações parasitárias (Poulin, 2011; Blaxter \& Denver, 2012; Poulin \& Randhawa, 2015). Encontra-se, principalmente, em trato digestivo e muitos deles estão relacionados a patologias clínicas diversas, enquanto outros não levam a nenhum tipo de efeito adverso (Kindlovits \& Kindlovits, 1999).

Os nematoides são a classe com a maior diversidade de ciclos de vida, podendo ser transmitidos em ciclos diretos pela ingestão de ovos, penetração cutânea, via lactogênica, ou ainda por transmissão intrauterina. Sua variedade inclui até espécies que podem também apresentar ciclos que incluem estágios em vida livre (Orihel \& Seibold, 1972; Cheng, 1974; Toft \& Eberhard, 1998). Algumas espécies de nematoides só ocorrem em primatas não humanos, porém, a maioria pode ocorrer em outros mamíferos, inclusive em seres humanos (Orihel \& Seibold, 1972). A patogenicidade das espécies de nematoides em seus hospedeiros também pode variar desde infecções assintomáticas à severos processos de enterites ulcerativas e hemorragias pulmonares.
No gênero Spirura sp. estes nematoides são vistos em região da parede esôfago, possui um aspecto definitivo de sua calda em espiral nos machos e suas fases de vida sua completadas em artrópodes, no quais são usados como hospedeiro intermediário (Urquhart, 1996). Este gênero, com sua diversidade de espécies, é identificado como de grande potencial de infecção parasitária em primatas brasileiros, isso leva ao aparecimento de doenças e é de suma importância para o sistema de estimativa na saúde ambiental, devido à influência resultante da intervenção humana. A correlação entre parasito e hospedeiro em animais silvestres de vida livre está bastante ligada às características peculiares do hospedeiro, como sua dieta, sua forma de viver, contatos sem especificidade, o que levou a perceber que quanto mais convivência mais carga parasitária e maior predominância de infecção e características externas como a desintegração do habitat, mudança de clima, heterogeneidade de insetos e plantas, tudo isso também atinge a estruturação da população de parasitos (Gillespie et al., 2005).

Pesquisas com amostras de fezes coletadas de Callithrix jacchus encontraram-se ovos de nematóides das famílias de Ancylostomatidae, Ascarididae, Physalopteridae, Strongilidae $e$ Trichuridae. Outros nematoides já foram encontrados e descritos em saguis-de-tufosbrancos como Dipetalonema gracilis, Filariopsis barretoi, Primasubulura distans, Primasubulura jacchi, Strongyloides sp., Trichospirura leptostoma, Trypanoxyuris callithricis. Os gêneros de primatas neotropicais mais acometidos são Alouatta, Sapajus libidinosus e Saimiri (Verona, 2008). No Brasil existem alguns relatos de parasitos Spirura sp. encontrados em saguis no estado do Rio de Janeiro (Verona, 2008) e no Pará (Moreira et al., 2012), em contrapartida, não são observados relatos deste parasito em Calitriquídeos na região Nordeste, sendo este o primeiro relato de Spirura sp. na região Nordeste.

\section{Considerações Finais}

O presente trabalho documenta um caso de infestação de parasito Spirura sp. em sagui-detufos-brancos e contribuem com conhecimento da importância do controle da transmissão de parasitas para assim elaborar as ações preservativas e epidemiológicas de zoonoses, já que há um aumento considerado do contato entre humanos e Callithrix jacchus. 


\section{Referências Bibliográficas}

Anderson, R.C., Chabaud, A.G., Willmott, S., 2009. Keys to the nematode parasites of vertebrates: archival volume. Commonwealth Agricultural Bureaux.

Blaxter, M., Denver, D.R., 2012. The worm in the world and the world in the worm. BMC Biology 10, 57.

Brack, M., 2012. Agents transmissible from simians to man. Springer Science \& Business Media, Bedin.

Carmo, A.M., Salgado, C.A., 2009. Ocorrência de parasitos intestinais em Callithrix sp. (Mammalia, Primates, Callithrichidae). Revista Brasileira de Zoociências 5, 267-272.

Cheng, T.C., 1974. Digenea - The Digenetic Trematodes. General Parasitology. Academy Press, pp. 371-463.

Gillespie, T.R., Chapman, C.A., Greiner, E.C., 2005. Effects of logging on gastrointestinal parasite infections and infection risk in African primates. Journal of Applied Ecology 42, 699707.

Kindlovits, A., Kindlovits, L.M., 1999. Clínica e terapêutica em primatas neotropicais. Universisdade Federal de Juiz de Fora, Juiz de Fora, Minas Gerais.

Moreira, V.L.C., Cordeiro, H.C., Nazaré, L.C., Giese, E.G., Maldonado Júnior, A., Santos, J.N., 2012. Nematofauna de Proechimys cf. roberti (Thomas, 1901) (Rodentia: Echimyidae), mamífero silvestre da região amazônica, Pará-Brasil. Parasitologia Veterinária, São Luis, Maranhão.

Orihel, T.C., Seibold, H.R., 1972. Nematodes of the Bowel and tissues. Pathology of Simian Primates Part II: Infectious and Parasitic Diseases. Fiennes, London.
Poulin, R., 2011. The many roads to parasitism: a tale of convergence. Advances in parasitology. Elsevier, pp. 1-40.

Poulin, R., Randhawa, H.S., 2015. Evolution of parasitism along convergent lines: from ecology to genomics. Parasitology 142, S6S15.

Rylands, A.B., 2012. Taxonomy of the Neotropical Primates - database. International Union for Conservation of Nature (IUCN), Species Survival Commission (SSC), Primate Specialist Group, Gland.

Toft, J.D., Eberhard, M.L., 1998. Parasitic Deseases. Nonhuman Primates in Biomedical Research Diseases. Academic Press, San Diego.

Urquhart, G.M., 1996. Parasitologia veterinária. Guanabara Koogan, Rio de Janeiro.

Verona, C.E.S., 2008. Parasitos em sagüi-de-tufobranco (Callithrix jacchus) no Rio de Janeiro. Fundação Oswaldo Cruz. Escola Nacional de Saúde Pública Sérgio Arouca, Rio de Janeiro, Brasil.

Vicente, J.J., Rodrigues, H.O., Gomes, D.C., Pinto, R.M., 1997. Nematóides do Brasil. Parte V\&58; nematóides de mamíferos Brazillan nematodes. Part V\&58; nematodes of mammals. Revista Brasileira de Zoologia 14, $1-452$.

\footnotetext{
Recebido: 26 Jun. 2018.

Aprovado: 14 Jul. 2018

Publicado: 16 Ago. 2018
}

Licenciamento: Este artigo é publicado na modalidade Acesso Aberto sob a licença Creative Commons Atribuição 4.0 (CC-BY 4.0), a qual permite uso irrestrito, distribuição, reprodução em qualquer meio, desde que o autor e a fonte sejam devidamente creditados. 